\title{
Specifying and Verifying Partial Order Properties Using Template MSCs ${ }^{\star}$
}

\author{
Blaise Genest ${ }^{1}$, Marius Minea ${ }^{2}$, Anca Muscholl ${ }^{1}$, and Doron Peled ${ }^{3}$ \\ 1 LIAFA, Université Paris VII \& CNRS \\ 2, pl. Jussieu, case 701475251 Paris cedex 05, France \\ 2 Department of Computing, "Politehnica" University of Timişoara \\ Bd. V. Pârvan nr. 2, RO-300223 Timişoara, Romania \\ 3 Department of Computer Science The University of Warwick \\ Coventry, CV4 7AL United Kingdom
}

\begin{abstract}
Message sequence charts (MSC) are a graphical language for the description of communication scenarios between asynchronous processes. Our starting point is to model systems using an assume-guarantee formalism, in the style of LSCs and Triggered MSCs. We enrich MSCs with the possibility of using gaps (template MSC), and show their expressivity. This formalism also allows to express logical formulas. We analyze the model-checking problem, whose complexity is linear in the size of the system, and ranges from PTIME to EXPSPACE in the size of the template formula.
\end{abstract}

\section{Introduction}

Concurrent systems are intricate and hence difficult to describe. The classical description, stemming from programming practices, is based on listing the different concurrent participants, e.g., the processes. The Message Sequence Charts (MSC) formalism allows an alternative "sequential" description of a concurrent system, where the complete behavior of all the processes involved in some given task are depicted in a visual way. The language enjoys widespread use in the specification of telecommunication protocols and has been standardized by the ITU-T [1. In a single MSC we can describe the behavior of all the processes involved, including the local actions and the messages exchanged between them. Such a slicing of the concurrent execution provides further intuition about the behavior of the system. One of the drawbacks of this representation is that tasks are seldom executed in a sequential way, and some overlap commonly exists.

In this paper we study an MSC-related formalism that allows expressing noncontiguous tasks. This is done by adding gaps to the MSC formalism. Intended for the analysis of systems, we present the formalism and study related verification problems. We are influenced in our proposal by Live Sequence Charts [4] and Triggered MSCs [16], and include an assume-guarantee mechanism, i.e., being able to require the execution of a task provided that another task was executed.

\footnotetext{
* Work supported by the EU-TMR project GAMES.
} 
While an individual MSC has a formally defined semantics, its relation to the system behavior is left open by the standard: the usual interpretation is that the scenario should be possible in the implementation. In defining Live Sequence Charts, Damm and Harel [4] extensively emphasize the duality of mandatory and provisional semantics, but with a much wider set of features, including abort/exit conditions and reliable or lossy transmission. The provisional semantics is used with the standardized High-Level MSCs (HMSCs for short), that are described by (hierarchical) graphs with nodes labeled by MSCs [1]. The semantics of an HMSC is the set of MSCs formed by concatenating (process by process) MSCs seen along a path. HMSCs have several drawbacks, such as the difficulty to express concurrency between two independent threads, due to the sequential control of the graph. The result is that many systems are hard to model using HMSCs. To address this problem, other kinds of specifications have been proposed, e.g. based on Petri nets with transitions labeled by MSCs [12].

A totally different approach is taken by Triggered MSCs [16. They replace the sequential description of HMSCs by an assume-guarantee formalism (that also exists in LSCs in form of activation messages). Causality is expressed by structuring a specification with two components: a precondition that identifies the initial behavior, and a postcondition expressing the continuation supposed to be guaranteed under this assumption. Assume-guarantee combined with the parallel operator emphasizes compositionality: a system description is most easily obtained combining MSCs for collections of directly interacting processes, and superimposing assume-guarantee patterns that further constrain interactions between individual scenarios.

We are inspired by the Triggered MSCs notation. Our suggestion attempts to improve on several points, for example, making the use of infinite assumeguarantee easier to understand. Our main contribution is to define template MSCs, and use them in the Triggered MSCs setting. We achieve conciseness by specifying only events strictly needed to identify a scenario and by using gaps as placeholders for other messages. With gaps, parallel composition can be simply expressed as conjunction, without the need for parallel (shuffle) operators. Using assume-guarantee template MSCs we can easily specify loops and thus infinite specifications.

A second important use of assume-guarantee template MSCs is the ability to easily specify properties that a system should satisfy (the system is given here as a set of FSMs communicating through (existentially) bounded FIFO message queues, or as an HMSC). We can express temporal properties, e.g. the fact that whenever $A$ happens, $B$ should eventually follow. Compared to temporal logics, MSCs have the advantage of being a visual formalism, and thus easier to use in a design and engineering environment. Moreover, template MSC formulas are a fragment of a partial-order global logics with filter, whose complexity would be much higher. We study the complexity of verifying temporal properties expressed by various classes of templates and show that it ranges from PTime to ExpSpace in the size of the formula, and is linear-time in the size of the system. 
One of the main differences between template MSCs and LSCs or Triggered MSCs is the use of gaps inside the MSC notation, in order to express an arbitrary (but finite) amount of communication or events. The user can also draw single send/receive events, with the matching event being located in a gap. Another difference is that we are using template MSCs as a visual specification formalism, as an alternative to temporal logic specification. Our specification is partial-order based, related to logics such as LTrL [175], TLC [3] and MSO [13.

A variant of model-checking for MSCs and HMSCs is considered in [15]. It uses an alternative semantics that consists in adding gaps between each pair of events on each process. This allows combating the undecidability of HMSC intersection. The approach in this paper is different. Gaps are added in the specification, and their locations and types need to be explicitly specified. For the full version see $h$ tp://www.crans.org/ genest/fossacs03_full_paper.ps.

\section{Message Sequence Charts and Templates}

Message Sequence Charts (MSC for short) is a scenario language standardized by the ITU, 1. They represent simple diagrams depicting the activity and communications in a distributed system. The entities participating in the interactions are called instances (or processes) and are represented by vertical lines. Message exchanges are depicted by arrows from the sender to the receiver. In addition to messages, atomic actions can also be represented.

The left part of Figure 1 gives an example of an MSC $M$ modeling two messages sent between a Writer $W$ and a Server $S$.

Definition 1 An $M S C$ is a tuple $M=\langle\mathcal{P}, E, \mathcal{A}, \ell, m,<\rangle$ where:

$-\mathcal{P}$ is a finite set of processes,

- E is a finite set of events,

- $\mathcal{A}$ is a finite set of names for messages and local actions,

- $\ell: E \rightarrow \mathcal{T}=\{p ! q(a), p ? q(a), p(a) \mid p \neq q \in \mathcal{P}, a \in \mathcal{A}\}$ labels an event with its type: in process $p$, either a send $p ! q(a)$ of message a to process $q$ (respectively, a receive $p ? q(a)$ of message a from process $q)$ or a local event $p(a)$. The labeling $\ell$ partitions the set of events by type (send, receive, or local), $E=S \cup R \cup L$, and by process, $E=\bigcup_{p \in \mathcal{P}} E_{p}$.

- $m: S \rightarrow R$ is a bijection matching each send to the corresponding receive. If $m(s)=r$, then $\ell(s)=p ! q(a)$ and $\ell(r)=p ? q(a)$ for some $p, q \in \mathcal{P}$ and $a \in \mathcal{A}$.

$-<\subseteq E \times E$ is an acyclic relation between events consisting of:

- a total order on $E_{p}$, for every process $p \in \mathcal{P}$, and

- $s<r$, whenever $m(s)=r$.

The event labeling $\ell$ implicitly defines the process $\operatorname{pr}(e)$ for each event $e$ : $\operatorname{pr}(e)=p$ if $e \in E_{p}$, i.e., $\ell(e) \in\{p ! q(a), p ? q(a), p(a)\}$ for some $q \in \mathcal{P}, a \in \mathcal{A}$. We assume that channels are FIFO, i.e., there is no overtaking on messages sent on the same channel.

The relation $<$ is called the visual order on the $\mathrm{MSC}$, since it corresponds to its graphical representation. It is comprised of the process ordering and the message ordering, pairwise between send and matching receive. 
Since $<$ is required to be acyclic, its reflexive-transitive closure $<^{*}$ is a partial order on the set $E$ of events, which we will denote by $\leq$. An extension of $\leq$ to a total order on $E$ is called a linearization of $M$. We denote by $\operatorname{Lin}(M)$ the set of all labeled linearizations of an $\operatorname{MSC} M, \operatorname{Lin}(M)=$ $\left\{\ell\left(e_{1}\right) \cdots \ell\left(e_{n}\right) \mid e_{1} \cdots e_{n}\right.$ is a linearization of $\left.M\right\}$.

The main objective in this paper is to model complex communication interactions using finite scenarios. We argue that HMSCs are hard to use in specification, since a designer must be able to describe the global behavior of a protocol in form of a graph. In general, finite MSCs are easier to use, since they capture the essence of scenarios. To describe one particular aspect of the system behavior, it is frequently not needed to consider all message exchanges or indeed not even all processes. Thus, we propose to use templates, which are diagrams in which events/messages can alternate with gaps. Gaps are effectively placeholders for arbitrary many (eventually zero) events or messages between designated processes. They can be instantiated by compositional MSCs (CMSC for short), that is an extension of MSCs [7]. The difference between a CMSC and an MSC is that the message function $m$ is a partial function, i.e., there can be sends or receives for which no matching event is defined. A send $s$ is called matched if the (matching) receive $r=m(s)$ is defined. By instantiating gaps by CMSCs, there can be messages exchanged between different gaps or messages composed by an event of the diagram and an event in a gap. The template MSC $N$ in the right part of Figure 1 describes the set of all CMSCs containing the message $a$.

Definition 2 (Template $M S C$ ) A template $M S C$ is a tuple $\langle\mathcal{P}, E, \Gamma, \mathcal{A}, \ell, m,<\rangle$, where $\langle\mathcal{P}, E, \mathcal{A}, \ell, m,<\rangle$ is a $C M S C$, with the following components extended:

- $\Gamma$ is a finite set of gap markers,

- $\ell: E \cup \Gamma \rightarrow \mathcal{T} \cup 2^{\mathcal{T}}$, with $\ell(\gamma) \subseteq \mathcal{T}$ the message types allowed in gap $\gamma \in \Gamma$. Let $\Gamma_{p} \subseteq \Gamma$ be the set of gaps $\gamma$ such that $\ell(\gamma)$ allows events on process $p$.

$-\leq \subseteq(E \cup \Gamma)^{2}$. For each process $p \in \mathcal{P}$ the restriction of $<$ to $E_{p} \cup \Gamma_{p}$ must be a total order.

The order between gaps and events in the above definition ensures that template MSCs can be effectively represented as diagrams. The semantics of a template MSC is an infinite set of CMSCs, obtained by replacing the gaps by CMSCs of allowed types.

Definition 3 (Semantics) A template $M S C M=\langle\mathcal{P}, E, \Gamma, \mathcal{A}, \ell, m,<\rangle$ defines a set of CMSCs, denoted by $\mathcal{L}(M)$. A CMSC $M^{\prime}=$ $\left\langle\mathcal{P}, E^{\prime}=E \cup \bigcup_{\gamma \in \Gamma} E^{\gamma}, \mathcal{A}, \ell^{\prime}, m^{\prime},<^{\prime}\right\rangle$ is in $\mathcal{L}(M)$ if it is obtained by replacing each gap $\gamma \in \Gamma$ with a (possibly empty) $C M S C M^{\gamma}$ with event set $E^{\gamma}$ such that:

- The type function $\ell^{\prime}$ is the union of $\ell$ and the type function of each $M^{\gamma}$. It is required that $\ell^{\prime}(e) \in \ell(\gamma)$ for every event $e \in E^{\gamma}$ (i.e., $M^{\gamma}$ contains only events of allowed types).

- The message function $m^{\prime}$ extends $m$ and the message function of each $M^{\gamma}$. It is required that $m^{\prime}$ preserves the FIFO restriction on matched events. 
- The visual order $<^{\prime}$ is the union of $<$, the visual order of each $M^{\gamma}$, and the set of all pairs $(e, f)$ satisfying $m^{\prime}(e)=f$ or $\operatorname{pr}(e)=\operatorname{pr}(f)$ and one of the following:

- $e \in E^{\gamma}, f \in E^{\kappa}$ with $\gamma<\kappa$ (both $e, f$ in gaps),

- $e \in E^{\gamma}, f \in E$ with $\gamma<f$ (e in a gap),

- $e \in E, f \in E^{\gamma}$ with $e<\gamma$ (f in a gap).

Remark. The ordering required between an event $e$ and a gap $\gamma$ sharing some process, does not imply an order between $e$ and all events of $\gamma$. That is, we may have two unordered events $e, f$ in $M^{\prime}$, with $f$ belonging to some gap $\gamma$ where $e<\gamma$ (however, $\operatorname{pr}(e) \neq \operatorname{pr}(f)$ in this case). Moreover, we may have $e<\gamma<f$ in $M$ with $e, f \in E, \gamma \in \Gamma$, but by replacing $\gamma$ with the empty MSC, $e, f$ are not ordered in $M^{\prime}$.

Note also that with our definition it is possible to obtain different message functions $m^{\prime}$ for the same instantiation $\left(M^{\gamma}\right)_{\gamma \in \Gamma}$ of gaps. This is needed since we will concatenate two such CMSC instantiations $M_{1}, M_{2}$, such that the result is an MSC. Thus the message function of $M_{2}$ depends on $M_{1}$.

Notice also that compositionality in gaps is mandatory if one wants e.g. to describe the set of all MSCs containing a given message. Assume for instance that in figure 1 the gaps of $N$ are instantiated by MSCs, and not by CMSCs. Then the MSC $M$ in the left part does not belong to the template $\mathcal{L}(N)$.

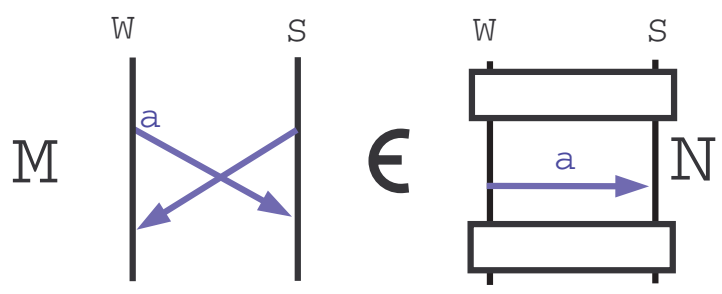

Fig. 1. Template $N$ representing all (C)MSCs containing the message $a$

We define $\operatorname{Lin}(M)$ for a template $\mathrm{MSC} M$ as the union of the linearizations of all CMSCs from $\mathcal{L}(M)$.

Template MSCs describe only simple communication patterns. To increase their expressivity, we can use them in an assume-guarantee framework, that allows in particular to express safety and liveness-like properties (see section 4.1). For defining assume-guarantee template MSCs we first define what it means to decompose an MSC $N$ as $N=S T$, where $S, T$ are CMSCs. It means that there exists a linearization $x_{1} \cdots x_{k}$ of $N$ and some $1 \leq i \leq k$ such that $x_{1} \cdots x_{i}$ $\left(x_{i+1} \cdots x_{k}\right.$, resp.) is a linearization of $S(T$, resp.).

Definition 4 (Assume-guarantee template $M S C s$ ) Let $M_{a}, M_{g}$ be two template MSCs. Then $M_{a} \longmapsto M_{g}$ and $M_{a} \longmapsto \neg M_{g}$ are assume-guarantee template MSCs that define sets of $M S C s$, denoted by $\mathcal{L}\left(M_{a} \longmapsto M_{g}\right), \mathcal{L}\left(M_{a} \longmapsto \neg M_{g}\right)$ : 
- $\mathcal{L}\left(M_{a} \longmapsto M_{g}\right)=\{N \in M S C \mid$ for every decomposition $N=S T$, either $S \notin \mathcal{L}\left(M_{a}\right)$ or $\left.T \in \mathcal{L}\left(M_{g}\right)\right\}$.

- $\mathcal{L}\left(M_{a} \longmapsto \neg M_{g}\right)=\{N \in M S C \mid$ for every decomposition $N=S T$, either $S \notin \mathcal{L}\left(M_{a}\right)$ or $\left.T \notin \mathcal{L}\left(M_{g}\right)\right\}$.

For an example of an assume-guarantee template MSC see Figure 4. Notice that $S, T$ can be CMSCs, but $S T=N$ is required to be an MSC. Note also that assume-guarantee template MSCs generalize MSCs, since every MSC $M$ can be represented as $\epsilon \longmapsto M$, where $\epsilon$ is the empty MSC.

A template MSC formula is a conjunction $\bigwedge_{i}\left(M_{a}^{i} \longmapsto\left(\bigvee_{j} \pm M_{g}^{i j}\right)\right)$, where \pm means that guarantee MSCs may appear in either positive or negated form. That is, for each of the individual assume-guarantee specifications of the outermost conjunction, we have preconditions in form of positive scenarios, and postconditions as disjunctions of either positive or negative (forbidden) scenarios. Hence, an MSC $N$ belongs to $\mathcal{L}\left(M_{a} \longmapsto \bigvee_{j} \pm M_{g}^{j}\right)$ if for every decomposition $N=S T$, whenever $S \in \mathcal{L}\left(M_{a}\right)$ either $T \in \mathcal{L}\left(M_{g}^{j}\right)$ for some positive $M_{g}^{j}$, or $T \notin \mathcal{L}\left(M_{g}^{j}\right)$ for some negative $M_{g}^{j}$. This conditional description allows in particular the guarantee false, with $\mathcal{L}($ false $)=\emptyset$. For example, an MSC $N$ satisfies $M \longmapsto$ false iff no prefix of $N$ is in $\mathcal{L}(M)$. The formula $\epsilon \longmapsto \neg M$ describes the complement of $\mathcal{L}(M)$.

\section{Modeling Using Template MSCs}

A first application of template MSCs is for modeling protocols easier than with HMSCs [1. The drawback of the standard notation of HMSCs is that one needs a global (graph) description combining several scenarios, resp. behaviors of the system. Using template MSCs, we model each behavior locally, that is each scenario is described on the processes that it involves. We restrict then the combination of these local behaviors by using template formulas. In the latter step, using template MSCs allows us to focus only on the relevant messages in a scenario, and avoid both repetition and the inclusion of unrelated messages.
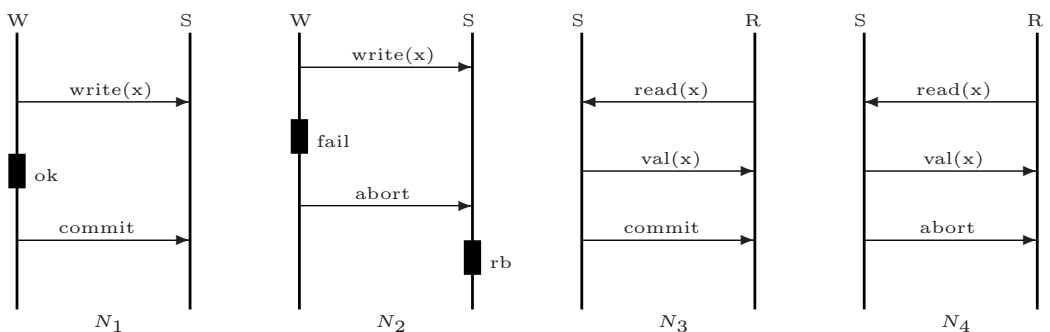

Fig. 2. Global Behavior of Writer-Server-Reader System: $\left(N_{1} \vee N_{2}\right)^{\star} \|\left(N_{3} \vee N_{4}\right)^{\star}$

We present an example that illustrates the major features of our approach, namely the reader-writer example, taken from [16]. The system consists of three 
processes: a writer $W$ and a reader $R$ which concurrently access variables maintained by a server $S$. The latter has the task of maintaining atomicity and serialization of read and write operations, each of which are performed in two phases. Since triggered MSCs cannot deal easily with infinite specifications, the example from [16] involves a single read/write operation. With template formulas we do not have this problem, so we extend this example to arbitrary many write/read operations.

The writer $W$ performs a tentative update of variable $x$ by sending a message $w(x)$ to the server $S ; x$ is now in a "dirty" state. Then, $W$ performs a local action ok or fail which decides on the outcome of the write, and sends the corresponding message commit or abort to the server. A commit marks the variable as "clean". An abort causes the server to perform a local rollback action $r b$ and potentially influences a read in progress. The reader $R$ can send the server a request $r(x)$ for the variable $x$, to which the server responds with a value $\operatorname{val}(x)$. Subsequently, the server either follows up with a commit message, if the sent value was clean, or has been since committed by the writer, or sends an abort if the sent value has to be rolled back. Although many different orders of interactions between the three processes are possible, the interaction between the pairs of directly communicating processes is simple. Our system description above contains a pair of basic scenarios for both writer-server and reader-server interaction, depicted in Figure 2

The global behavior is a subset of that given by composing these individual scenarios. Using a notation similar to Triggered MSCs, we would write $\left(N_{1} \vee N_{2}\right)^{\star}||\left(N_{3} \vee N_{4}\right)^{\star}$. However, one side effect of gaps is that they make the definition of a parallel composition operator unnecessary, assuming that we compare MSC with different type sets. To express $N_{1} \| N_{3}$ for instance, it suffices to extend both MSCs to all three processes, and add gaps in between all messages. The gaps in $N_{1}$ (resp. $N_{3}$ ) allow only events of $N_{3}$ (resp. $\left.N_{1}\right)$. Then, parallel composition simply becomes conjunction (language intersection): $\mathcal{L}\left(N_{1} \| N_{3}\right)=\mathcal{L}\left(N_{1}\right) \cap \mathcal{L}\left(N_{3}\right)$. We need slightly more work for expressing the star of languages. First, we need an initialization step $\left(\epsilon \longmapsto M_{1} \vee M_{2}\right)$ for the writer, meaning that every MSC in the specification should begin on $W, S$ by a write, and then either ok and commit, or fail and abort. Anything can happen next, as allowed by the unrestricted gap $\gamma_{\star}$. The MSCs $M_{1}, M_{2}$ are defined in figure 3 where the gap $\gamma_{\star}$ has no restriction, while $\gamma_{R}$ is restricted to events of $N_{3}, N_{4}$.

By adding an inductive step we obtain the specification. Namely, we need that either $M_{1}$ or $M_{2}$ happens after each message commit (or event $r b$ ), or there is no more event on $W, S\left(\right.$ gap $\left.\gamma_{R}\right)$, specified as:

$$
\gamma_{\star} \text { commit } \longmapsto M_{1} \vee M_{2} \vee \gamma_{R} \wedge \gamma_{\star} r b \longmapsto M_{1} \vee M_{2} \vee \gamma_{R}
$$

The same applies for $M_{3}, M_{4}$. These individual scenarios are interdependent, so the global system behavior is obtained by imposing additional constraints on their composition. We divide the constraints into an assumption part that identifies the initial behavior in a scenario, and a guarantee part expressing the behavior expected of the system under this assumption. 

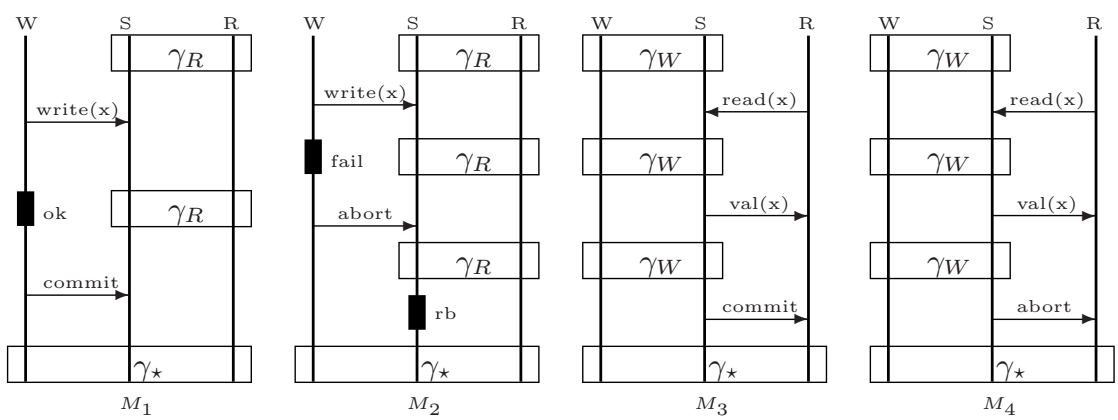

Fig. 3. Initialization: $\epsilon \hookrightarrow\left(M_{1} \vee M_{2}\right) \wedge \epsilon \succ\left(M_{3} \vee M_{4}\right)$

For our WSR example we identify 5 cases specified with the constraints in Figure 4. $M_{5}$ states that if a write on the Server is followed by a send of $x$ to the Reader, and the Writer aborts (precondition), then the Server should inform the Reader about the abort (postcondition). The occurrence of the read is guaranteed by $M_{3}$ or $M_{4}$, so it needs not be specified again. Likewise, $M_{1}, M_{2}$ ensure that if there is no write between a send of $x$ and an abort of the Writer, then the write has occurred in the first gap. This precondition will imply an

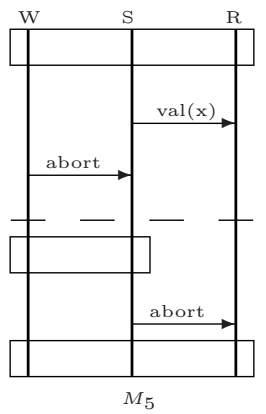
abort for the read (postcondition). The remainder of the interaction needs not be specified, so we allow gaps in between these actions, corresponding to sending and receiving other messages. The other cases correspond to a commit of the value. Namely, a value is sent while no write has been produced $\left(M_{6}\right)$, or a value is sent after the last write has been roll-backed $\left(M_{7}\right) /$ committed $\left(M_{8}\right)$, or a commit is received immediately after the value is sent $\left(M_{9}\right)$.
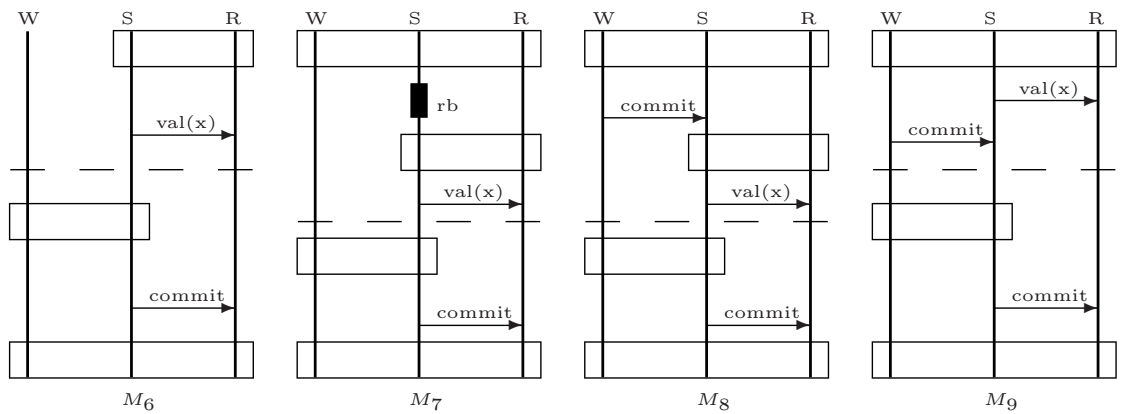

Fig. 4. Assume-Guarantee Scenarios for Writer-Server-Reader System

Hence the constraint is $M_{5} \wedge M_{6} \wedge M_{7} \wedge M_{8} \wedge M_{9}$. Without template MSCs, we would need to write at least every possible instantiation for gaps in our 5 cases, yielding at least 12 cases. For instance, an HMSC specifying the same 
model would require at least 19 states. Moreover, the size increases even more severely (exponentially) if instead of a single reader we allow several ones. With template formulas we express the constraints for each pair Writer/Reader, while an equivalent HMSC has to describe all possible combinations over all Readers. This lack of conciseness of HMSCs is a real drawback, since many algorithms involving MSCs are at least NP-hard. First, HMSCs are unable to represent the parallel composition, which can lead to an exponential blow-up compared with template formulas, and to specifications that are harder to understand. Second, HMSCs are finitely generated, which prevents them from implementing simple protocols such as the alternating bit. Third, HMSCs cannot be complemented in general. Hence, since template formulas implicitly complement the assume part they are not subsumed by HMSCs.

\section{Specifying Properties}

\subsection{Logical Properties}

Template MSC formulas can describe easily and in a concise way some interesting properties and can be model-checked (see next section). We can use them for describing global properties of MSC configurations and use gaps as filters, i.e., for restricting the types of events. We denote in the examples below by $\gamma$ an unrestricted gap over all processes, and by $\gamma_{\neg a}$ a gap that can generate all event types except for $a$.

$-(\gamma A) \longmapsto$ false $=\epsilon \longmapsto \neg(\gamma A \gamma)$ : No execution contains the MSC $A$.

$-\gamma \longmapsto \gamma A \gamma$ : Every execution contains infinitely often the MSC $A$.

- $\gamma A \longmapsto \gamma B \gamma$ : Whenever $A$ occurs, eventually $B$ will occur.

$-(\gamma A \longmapsto \gamma a \gamma) \wedge\left[\epsilon \longmapsto\left(\gamma_{\neg a} \vee \gamma_{\neg a} A \gamma\right)\right]$ : The MSC $A$ may occur. If this is the case, then the event $a$ must follow. Moreover, event a cannot occur before $A$. One can see $a$ as an alarm event that is triggered by $A$.

The theorem below shows that the expressiveness of compositional gaps has a drawback, namely that the satisfiability problem for template formulas is undecidable in general. However, we can check the satisfiability of a template formula $S$ if we ask only for MSCs that have at least one linearization where the size of each channel is bounded by some given value $b$ (it is possible that other equivalent linearizations have higher bounds). A set $S$ of such MSCs is called existentially $b$ bounded. For instance, every HMSC (even every realizable compositional HMSC, see [7]) is existentially bounded.

Theorem 1 1. Given a bound $b$ and a template $M S C$ formula $S$, it is decidable whether there exists an existentially b-bounded $M S C$ in $\mathcal{L}(S)$.

2. It is undecidable whether a template $M S C$ formula $S$ satisfies $\mathcal{L}(S) \neq \emptyset$.

The proof of the first statement above follows from the results in the next section. For the second statement, we reduce from the Post correspondence problem, making use of the unbounded communication channels. 


\subsection{Model-Checking Template Formulas}

We consider now the problem of verifying an implementation of a communication protocol $S$ with respect to a template MSC formula $\Phi$. A different approach using partial order MSO for the specification $\Phi$ gives decidability for the modelchecking problem [13], albeit at very high costs. As suggested by Theorem 1, the system $S$ needs an existential bound on buffers, denoted by $b_{S}$. This includes protocols modeled by HMSCs, communicating finite state machines with existentially bounded FIFO buffers (and even realizable compositional HMSCs, see [7]). The model for the implementation here is a finite automaton (FSM), generating linearizations of MSCs. We do not require that $S$ is linearization-closed, i.e., $S$ may generate a linearization of some MSC without generating all of them. We can obtain a linear-size FSM from any (realizable compositional) HMSC. It suffices to replace each node by a linearization of the CMSC labeling the node.

Definition 5 For an FSM $S$ and an assume-guarantee $M S C M_{a} \longmapsto \pm M_{g}$ we write $S \models\left(M_{a} \longmapsto \pm M_{g}\right)$ if $\mathcal{L}(S) \subseteq \mathcal{L}\left(M_{a} \longmapsto \pm M_{g}\right)$. The satisfaction of a template formula is defined according to the usual semantics of $\wedge, \vee$.

In the following, we give complexity results for checking $S \models \Phi$ for various classes of template MSC formulas $\Phi$. While $S$ can be very large, real life formulas $\Phi$ and existential channel bounds $b_{S}$ are pretty small. Hence we focus on keeping the complexity linear w.r.t. $S$. We will transform the formula into an automaton, so our algorithm will be automata-based. Moreover, checking that $S \models \bigwedge_{i} \Phi_{i}$ is done for each $\Phi_{i}$ separately.

Proposition 1 Given an FSM $S$ with channel bound $b_{S}$ and a template $M S C$ $M_{g}$, we can check whether $S \models \epsilon \longmapsto M_{g}$ in space exponential in $b_{S}\left|M_{g}\right|$ and logarithmic in $|S|$.

Proof. Let $E$ be the set of events in the template MSC $M_{g}$. Let $M$ be the MSC obtained from $M_{g}$ by replacing each gap by the empty MSC. Let us fix a linearization $x=x_{1} \cdots x_{n}$ of $M$. We show how to construct an NFA $\mathcal{A}_{x}$ accepting every linearization of $\operatorname{Lin}\left(M_{g}\right)$ whose events occur in the order given by $x$.

For each gap $\gamma$ of $M_{g}$ and each process $p$ that is allowed in $\gamma$ we use a new symbol $\gamma^{p}$. We first set the beginning and the end of $\gamma$ on process $p$ by choosing two positions $i \leq j$ in $x$ and then inserting one occurrence of $\gamma^{p}$ between each $x_{k}, x_{k+1}$ for $i \leq k<j$. The choice of $i, j$ must be consistent with the position of gap $\gamma$ on process $p$. For instance, both the events on $p$ before $\gamma$ and the symbols $\kappa^{p}$ with $\kappa<\gamma$ must precede $x_{i}$. Let $y$ be a string obtained in this manner. It remains to replace each symbol $\gamma^{p}$ by $X_{\gamma, p}^{*}$, with $X_{\gamma, p} \subseteq \ell(\gamma)$ the event types of $\gamma$ on process $p$. In order to obtain all linearizations of $\operatorname{Lin}\left(M_{g}\right)$, between any two consecutive events $x_{i}, x_{i+1}$, the NFA will generate all possible orderings of events that preserve the sequence of gaps $X_{\gamma, p}^{*}$ on process $p$. For instance, suppose that we have $X_{\gamma_{1}, p}^{*} X_{\gamma_{3}, r}^{*} X_{\gamma_{2}, p}^{*} X_{\gamma_{1}, q}^{*}$ between two events. Then the NFA generates $\left(X_{\gamma_{1}, p} \cup X_{\gamma_{3}, r} \cup X_{\gamma_{1}, q}\right)^{*}\left(X_{\gamma_{3}, r} \cup X_{\gamma_{2}, p} \cup X_{\gamma_{1}, q}\right)^{*}$. 
We have to ensure that messages of $M$ are preserved. For this, we use one counter (of maximal value $b_{S}$ ) per message in $M$. Furthermore, to ensure that a message is never received before being sent, we use one counter per communication channel (of maximal value $b_{S}$ ). When a message $m$ from $p$ to $q$ of $M$ is sent, the counter $C_{m}$ is initialized at 0 . We increment it when a message is sent from $p$ to $q$, and decrement when $q$ receives from $p$. When the message $m$ is received, we block the counter $C_{m}$. In the same way, we increment and decrement the counter corresponding to some channel, and block whenever it has value -1 . The automaton accepts iff all counters are 0 .

The resulting NFA is exponential in $b_{S}\left|M_{g}\right|$ and accepts all $b_{S}$-bounded linearizations of $\operatorname{Lin}\left(M_{g}\right)$. For a positive guarantee we can thus check $\mathcal{L}(S) \subseteq$ $\operatorname{Lin}\left(M_{g}\right)$ in space exponential in $b_{S},\left|M_{g}\right|$. For a negative guarantee we check $\mathcal{L}(S) \cap \operatorname{Lin}\left(M_{g}\right)=\emptyset$ in polynomial space in $b_{S},\left|M_{g}\right|$.

We can construct the same automaton for the precondition $M_{a}$. Then we compute in polynomial space the states of $S$ that can be reached from an initial state by some execution corresponding to an MSC in $\mathcal{L}\left(M_{a}\right)$. We obtain:

Theorem 2 Checking $S \mid=M_{a} \longmapsto \bigvee_{i}( \pm) M_{g}^{i}$ is in EXPSPACE $\left(b_{S}\left|M_{g}\right|\right)$ (and $\operatorname{PSPACE}\left(\left|M_{a}\right|\right)$. Checking $S \models M_{a} \longmapsto \bigvee_{i} \neg M_{g}^{i}$ is in PSPACE $\left(b_{S}\left|M_{g}\right|\left|M_{a}\right|\right)$.

\subsection{Model-Checking in PTIME}

While template specifications are quite expressive, we have seen in the previous section that model-checking is rather expensive. On the other hand, partialorder logics are in general more expensive than linear logics (e.g., LTrL is nonelementary [18]. For a natural fragment of LTrL that is related to our template formulas, where the until operator is replaced by the existential diamond operator, model-checking is also EXPSPACE, 318. In this section we consider a reasonable restriction of template MSCs that yields a polynomial time modelchecking algorithm. Basically, we require that 1 ) the guarantee template has only one gap, 2) the system is linearization-complete and 3) gaps must be instantiated by simple MSCs (instead of CMSCs).

Proposition 2 Checking $S \models \epsilon \succ M_{g}$, where $S$ is an FSM and $M_{g}$ is an MSC with at most one gap can be done in time polynomial in $\left|M_{g}\right|$.

Proof. For each process $p$, we show how to build an automaton $A_{p}$ of polynomial size, recognizing linearizations where the projection on $p$ contradicts the projection of $\operatorname{Lin}\left(M_{g}\right)$ onto $p$, denoted as $\prod_{p}\left(\operatorname{Lin}\left(M_{g}\right)\right)$. We have $\prod_{p}\left(\operatorname{Lin}\left(M_{g}\right)\right)=a_{1} \ldots a_{l} X_{p}^{*} b_{1} \ldots b_{m}$ where $X_{p}$ is the set of all events allowed in the unique gap of $M_{g}$ on process $p$. For generating $\mathcal{L}\left(A_{p}\right)=\overline{\prod_{p}\left(\operatorname{Lin}\left(M_{g}\right)\right)}$, we just need the disjunction of three regular sets, the first one testing that the number of events on $p$ is less than $l+m$, the second one recognizing the violation of the prefix $a_{1} \ldots a_{l}$ on $p$, and the third one recognizing the violation of the suffix $b_{1} \ldots b_{m}$ on $p$. Clearly, $\operatorname{Lin}(S) \cap \operatorname{Lin}\left(\bigcup_{p} A_{p}\right)=\emptyset$ iff $S \models \epsilon \longmapsto M_{g}$.

For model-checking vs. an assume-guarantee template MSC in polynomial time we consider only linearization-complete systems. Recall that a system $S$ is 
linearization-complete if for each execution of $S$ that is the linearization of an MSC $M$, every linearization of $M$ is an execution of $S$. Such a system can be derived from a regular MSC language [10.

Proposition 3 Given a linearization-complete system $S$ and a template $M S C$ $M_{a}$, we can construct a polynomial-size NFA A such that:

1. $\mathcal{L}(A) \cap \mathcal{L}(S) \subseteq \operatorname{Lin}\left(M_{a}\right) \cap \mathcal{L}(S)$, and

2. for each $M S C M \in \mathcal{L}\left(M_{a}\right)$ with $\operatorname{Lin}(M) \cap \mathcal{L}(S) \neq \emptyset$, A accepts at least one linearization in $\operatorname{Lin}(M) \cap \mathcal{L}(S)$.

In particular, the last proposition implies that we can determine in polynomial time the states of $S$ that can be reached from an initial state by some execution corresponding to an MSC in $M_{a}$, by computing $\mathcal{L}(A) \cap \mathcal{L}(S)$.

Proof. Consider an arbitrary linearization $x_{1} \cdots x_{n}$ of $M_{a}$, with $x_{i} \in E \cup \Gamma$. Since $S$ is complete, any MSC in $\mathcal{L}\left(M_{a}\right)$ that has a linearization in $\mathcal{L}(S)$ also has a linearization in $\mathcal{L}(S)$ corresponding to $x_{1} \cdots x_{n}$. We construct $A$ as a sequencing of NFA for each $x_{i}$. For events, the NFA is trivial, with one transition. For gaps, we note that any event sequence in $A$ which doesn't respect the message order is eliminated on intersection with $S$. Thus, it suffices to build for each gap an NFA which at each state has a transition for any event $e \in \ell(\gamma)$, augmented with incrementing a global counter on each send (and decrementing it on each receive). The NFA accepts when the counter is zero, i.e. the number of total sends and receives is balanced. The intersection with $S$ ensures acceptance of only those sequences which are balanced for each individual message. The value of the counter is bounded by the total number of outstanding messages, which is less than the size of $S$. Thus, $A$ is of polynomial size.

Notice that we cannot check a template formula $M_{a} \longmapsto \bigvee_{j} M_{g}^{j}$ in PTIME since the non-emptiness problem of the intersection of several automata is already PSPACE-hard. We can just generalize to the following:

Theorem 3 Checking that $S \models \bigwedge_{i}\left(M_{a}^{i} \longmapsto\left( \pm M_{g}^{i}\right)\right)$ where $S$ is a linearizationcomplete system, each $M_{g}^{i}$ has at most one gap and gaps in both $M_{a}^{i}, M_{g}^{i}$ must be instantiated by MSCs, can be done in time polynomial in the size of $M_{a}^{i}, M_{g}^{i}$ and $S$.

\subsection{Closing the Gap between PTIME and EXPSPACE}

A natural question arising now is which special cases of assume-guarantee template MSCs can be model-checked in less than exponential space. This is more than just a theoretical question, since model-checking in the general setting is very expensive, while the PTIME case given in section 4.3 can only express very simple properties.

In this section we restrict the guarantee template to have at most two gaps, which basically means pattern matching a finite MSC. Notice that even the complex alarm property in section 4.1is rather concise, it uses just two gaps. 
The main result given in this section is that model-checking assume-guarantee template MSCs where the guarantee part has at most two gaps, can be done in PSPACE. Since the guarantee part is usually small, a polynomial space algorithm in the size of the formula and logarithmic in the size of the system is feasible in practice (remember that model checking an FSM against an LTL formula is also polynomial space in the size of the formula, but logarithmic in the size of the FSM).

Proposition 4 Consider an FSM S (linearization-complete or not), and a template $M S C M$ with 2 gaps. Checking that $S \models \epsilon \longmapsto M$ is in $\operatorname{PSPACE}\left(b_{S}|M|\right)$.

We describe the algorithm first for an easier case, namely when $M=\gamma P \gamma$, where $\gamma$ is a gap on all processes without any type restriction, and $P$ is a finite MSC (pattern). We adapt the pattern matching algorithm of [8] for Mazurkiewicz traces, improving the result stated for hierarchical MSCs in 6]. Intuitively, we do string pattern matching for each projection $P_{p}$ of the pattern $P$ on a process $p$ and then determine occurrences that match together to an MSC pattern.

Formally, for an MSC $N$ and a process $p$ we denote by $N_{p}$ the sequence of events of $N$ on $p$. The idea is to compute for each process $p$ the positions $x_{p}$ of $M_{p}$ where $P_{p}$ occurs, and check that there exists some tuple of positions $\left(x_{p}\right)_{p \in \mathcal{P}}$ that corresponds to the pattern $M$. We locate a pattern $P_{p}$ immediately after its last event. For simplicity, if $P$ has no event on process $p$, then we do as if there is a pattern $P_{p}$ after any event of $M$ on $p$.

Definition 6 Let $x_{p}, x_{q}$ be two occurrences of $P_{p}$ and $P_{q}$, resp., in the $M S C M$. We call $x_{p}, x_{q}$ compatible if 1$)$ there is no message $(s, r)$ in $M$ from $p$ to $q$ with $x_{p}<s<r<x_{q}$ and no message from $q$ to $p$ with $x_{q}<s<r<x_{p}$, and 2) if $P$ contains a message from $p$ to $q$, then there is no message $(s, r)$ in $M$ from $p$ to $q$ with $s<x_{p}$ and $x_{q}<r$.

The next proposition states that the compatibility relation suffices for knowing whether $M=\gamma P \gamma$, i.e., whether $P$ occurs in $M$ or not.

Proposition 5 A $\wp$-tuple $\left(x_{p}\right)_{p \in \mathcal{P}}$ is an occurrence of $P$ in an $M S C M$ iff $x_{p}, x_{q}$ are compatible for all $p, q$.

Proof. The implication from left to right follows easily. For the converse assume by contradiction that $\left(x_{p}\right)_{p \in \mathcal{P}}$ is not a pattern because for some pair of processes $p, q$, the sends from $p$ to $q$ do not match the receives of $q$ from $p$.

There are several cases to consider. Either $x_{p}$ ends before the send of the last message $m$ from $p$ to $q$ that hits $x_{q}$, and then this message $m$ is after $x_{p}$ and before $x_{q}$, which is not possible. Or $x_{q}$ ends before the receive of the last message $m$ from $p$ to $q$ that was issued in $x_{p}$, and then this message $m$ is before $x_{p}$ and after $x_{q}$, which is not possible by the additional rule.

The last case where $\left(x_{p}\right)_{p \in \mathcal{P}}$ is not a pattern is because of some chain of messages $\left(s_{k}, r_{k}\right)_{1 \leq k \leq m}$ with $p_{k}=\operatorname{pr}\left(s_{k+1}\right)=\operatorname{pr}\left(r_{k}\right), r_{k}<s_{k+1}$ for all $k$, and such that $p_{1}=p, p_{m+1}=q, x_{p}<s_{1}, r_{m}<x_{q}$. Thus, there exists some $k$ 
such that $x_{p_{k}}<s_{k}$ and $r_{k}<x_{p_{k+1}}$. But this means that $\left(x_{p_{k}}, x_{p_{k+1}}\right)$ are not compatible, a contradiction.

The overall idea is to generate on-the-fly every pattern $x_{p}$ for all $p$, and to compute the last pattern $x_{p}$ can be compatible with. If some $x_{p}$ is not compatible with any $x_{q}$ (for at least one $q$ ), then we delete it. For instance, let $(s, r)$ be a message between $p$ and $q$, and $X_{q}$ be the last pattern on $q$ before the receive $r$. For all patterns $x_{p}<s$ on process $p$, the pattern $X_{q}$ is the last pattern $x_{p}$ can be compatible with, since any pattern $x_{q}>r$ on $q$ would satisfy $x_{p}<s<r<x_{q}$. In the case where $X_{q}$ does not exist, $x_{p}$ cannot be matched and should be deleted.

Let $A$ be the automaton corresponding to the product of deterministic automata recognizing (word) patterns on each process, using the pattern matching algorithm of Knuth-Morris-Pratt.

We build an automaton $B$ based on $A$ that recognizes runs (linearizations) that do not contain pattern $P$. The states of $B$ are of the form ( $a, S$, Pattern, $c)$, where

- $a$ is a state of $A$.

$-S$ is the set of unmatched sends seen so far.

- Pattern $=\bigcup_{p}$ Pattern $_{p}$, where $\operatorname{Pattern}_{p}$ is the set of patterns on process $p$ (not deleted) seen so far.

$-c$ is the compatibility function. For $x \in$ Pattern, $p \in \mathcal{P}, c(x, p) \in \operatorname{Pattern}_{p} \cup$ $\{+\infty\}$ is the last pattern $x$ is compatible with. It equals $+\infty$ if $x$ is compatible with any pattern on $p$.

\begin{tabular}{|c||c|c|}
\hline$S \models M_{a} \longmapsto M_{g}$ & $M_{a}$ & $M_{g}$ \\
\hline \hline PTIME & $\begin{array}{c}\text { closed gaps and S complete } \\
\text { or one closed gap }\end{array}$ & $\begin{array}{c} \pm \text { one closed gap, } \\
\text { no disjunction }\end{array}$ \\
\hline PSPACE & no restriction & negative templates or \pm two gaps \\
\hline EXPSPACE & no restriction & no restriction \\
\hline
\end{tabular}

Fig. 5. Complexity of Model-Checking

We describe now the transitions. Let $e$ be an event. Then $(a, S$, Pattern, $c) \rightarrow_{e}$ $\left(a^{\prime}, S^{\prime}\right.$, Pattern' $\left.{ }^{\prime} c^{\prime}\right)$ iff $a \rightarrow_{e} a^{\prime}$ in $A$ and

1. if $e=p ! q$ then Create_new_send $(e)$

2. if $e=p ? q$, let $s \in S$ matching the receive $e$. Update_dependencies $(s, e)$

3. if $\operatorname{pr}(e)=p$ and $A$ recognizes a pattern on $p$, Create_new_pattern $(p)$.

Proposition 6 Let $B$ have final states of the form ( $a, S=\emptyset$, Pattern, $c)$, such that Pattern $_{p}=\emptyset$ for at least some process $p$. Then $M \in \mathcal{L}(B)$ iff $P$ does not occur in $M$.

For the general case we have to consider a guarantee template MSC of the form $M_{g}=M_{1} \gamma_{T} P \gamma_{T^{\prime}} M_{2}$, where $T, T^{\prime}$ are sets of event types, and $M_{1}, P, M_{2}$ 
are CMSCs. We deal with this case by extending the automaton $B$ in a suitable way, and combining with the ideas of section 4.3 .

Theorem 4 Let $S$ be an FSM, $M_{g}=\bigvee_{i}( \pm) M_{g}^{i}$ be a guarantee template $M S C$ with at most two gaps and let $M_{a}$ be a template $M S C$.

Checking $S \models M_{a} \longmapsto \bigvee_{i}( \pm) M_{g}^{i}$ is PSPACE-complete in $\left(b_{S}\left|M_{g}\right|\left|M_{a}\right|\right)$.

\section{Conclusion}

We proposed template MSC formulas as an extension of Triggered MSCs by adding gaps and showed how to use them as a visual specification formalism. The two main components are the use of assume-guarantee CMSC as in Triggered MSCs and LSCs, together with gaps. The formalism is quite expressive and allows to specify safety and liveness-like properties. A drawback of the expressivity is that satisfiability is undecidable, unless there is an existential bound on communication channels. Notice that for LSCs, satisfiability (consistency) is shown to be decidable in [9] with a synchronous semantics of communication. However, synchronous communication is a severe restriction for specifying protocols.

We considered template MSCs as a specification formalism, given as a visual alternative to linear temporal logic, and we analyzed the complexity of checking various template MSC properties. Model-checking a realizable compositional HMSC $S$ gives the following complexities for the restrictions below:

\section{References}

1. ITU-TS recommendation Z.120, Message Sequence Charts, Geneva, 1999.

2. R. Alur and K. Etessami, and M. Yannakakis. Realizability and verification of MSC graphs. In ICALP'01, LNCS 2076, pp. 797-808, 2001.

3. R. Alur and K. McMillan and D. Peled. Deciding global partial-order properties In ICALP'98, LNCS 1443, pp. 41-52, 1998.

4. W. Damm and D. Harel. LSCs: Breathing life into Message Sequence Charts. Formal Methods in System Design 19(1): 45-80 (2001).

5. V. Diekert, P. Gastin. LTL is expressively complete for Mazurkiewicz traces. In JCSS 64(2): 396-418, 2002

6. B. Genest, A. Muscholl. Pattern Matching and Membership for Hierarchical Message Sequence Charts. In LATIN'02, LNCS 2286, pp. 326-340, 2002.

7. E. Gunter, A. Muscholl, and D. Peled. Compositional Message Sequence Charts. In $T A C A S^{\prime} 01$, pp. 496-511. LNCS 2031, 2001.

8. H. Liu, C. Wrathall and K. Zeger. Efficient Solution of Some Problems in Free Partially Commutative Monoids. Inf. and Comp., 89:180-198, 1990.

9. D. Harel and H. Kugler. Synthesizing State-Based Object Systems from LSC Specifications. In International Journal of Foundations of Computer Science 13(1): 5-51, 2002.

10. J. G. Henriksen, M. Mukund, K. Narayan Kumar, and P. Thiagarajan. On message sequence graphs and finitely generated regular MSC languages. In ICALP'O0, LNCS 1853, pp 675-686, 2000. 
11. P. Madhusudan. Reasoning about sequential and branching behaviours of message sequence graphs. In ICALP'01, LNCS 2076, pp. 809-820, 2001.

12. M. Mukund, K. Narayan Kumar, and P.S. Thiagarajan. Netcharts: bridging the gap between HMSCs and executable specifications. In CONCUR'03, LNCS 2761, pp 296-310, 2003.

13. P. Madhusudan, and B. Meenakshi. Beyond Message Sequence Graphs. In FSTTCS'01, LNCS 2245, pp 256-267, 2001.

14. A. Muscholl and D. Peled. Message sequence graphs and decision problems on Mazurkiewicz traces. In MFCS'99, LNCS 1672, pp. 81-91, 1999.

15. A. Muscholl, D. Peled, and Z. Su. Deciding properties for Message Sequence Charts. In FoSSaCS'98, LNCS 1378, pp. 226-242, 1998.

16. B. Sengupta and R. Cleaveland. Triggered Message Sequence Charts. In SIGSOFT 2002/FSE-10, ACM Press, 2002.

17. P.S. Thiagarajan and I. Walukiewicz. An expressively complete linear time temporal logic. In Information and Computation, 179(2): 230-249, 2002.

18. I. Walukiewicz. Difficult configurations - On the complexity of LTrL. In ICALP '98, LNCS 1443, pp. 140-151, 1998. 